\title{
Excess cause-specific mortality in in-patient-treated individuals with personality disorder: 25-year nationwide population-based study*
}

\author{
Emma Björkenstam, Charlotte Björkenstam, Herman Holm, Bengt Gerdin and Lisa Ekselius
}

\section{Background}

Although personality disorders are associated with increased overall mortality, less is known about cause of death and personality type.

\section{Aims}

To determine causes of mortality in ICD personality disorders.

\section{Method}

Based on data from Swedish nationwide registers, individuals admitted to hospital with a primary diagnosis of personality disorder between 1987 and 2011 were followed with respect to mortality until 31 December 2011. Standardised mortality ratios (SMRs) with 95\% confidence intervals and underlying causes of death were calculated.

\section{Results}

All-cause SMRs were increased, overall and in all clusters, for natural as well as unnatural causes of death. The overall
SMR was 6.1 in women and 5.0 in men, as high as previously reported for anorexia nervosa, with higher rates in cluster $\mathrm{B}$ and mixed/other personality disorders. The SMR for suicide was 34.5 in women and 16.0 in men for cluster B disorders. Somatic and psychiatric comorbidity increased SMRS.

\section{Conclusions}

The SMR was substantially increased for all personality disorder clusters. Thus, there was an increased premature mortality risk for all personality disorders, irrespective of category.

\section{Declaration of interest}

H.H. has participated on an advisory board for Janssen-Cilag AB. L.E. participates on advisory boards for $\mathrm{H}$. Lundbeck A/S and Eli Lilly Sweden AB.

\section{Copyright and usage}

(c) The Royal College of Psychiatrists 2015.
Personality disorders constitute a global health problem and are estimated to affect more than $5 \%$ of adults globally. ${ }^{1}$ They have a number of common features as well as diametrically different characteristics, and are organised into 10 categories in DSM-IV ${ }^{2}$ and eight in ICD-10. ${ }^{3}$ Based on clinical utility and symptoms, they are grouped into three clusters in DSM-IV: cluster A characterised by odd and eccentric behaviours, cluster B by dramatic and erratic behaviours and cluster $\mathrm{C}$ by anxious, fearful behaviours. In addition, a substantial number of individuals have disorders of personality functioning that do not fulfil criteria for a defined personality disorder. It is well established that individuals with a personality disorder have a higher mortality rate and a shorter life expectancy compared with the general population. ${ }^{4-9}$ In particular, there is an increased risk for death as a result of homicide, ${ }^{10}$ suicide or accidents. ${ }^{9,11}$ Comorbidity with other psychiatric ${ }^{12,13}$ as well as somatic conditions ${ }^{14}$ is common. It has been shown that having a cluster B personality disorder imposes an increased risk for cardiovascular death, ${ }^{15}$ and personality disorders in all clusters impose an increased risk for suicide. ${ }^{16}$ In general, however, it is not known whether the increased mortality in personality disorder is related to comorbidity with other psychiatric or somatic diseases rather than to the personality disorder as such, or how the increased mortality is distributed among the different personality disorders. Therefore, in this study we used nationwide Swedish registers to determine both overall as well as cause-specific standardised mortality ratios (SMR) in all individuals admitted to hospital for personality disorder during a period of up to 25 years. Results are reported for each cluster and for women and men separately. In addition, we analysed to what extent somatic and

*Presented in part at the 166th American Psychiatric Association Annual Meeting 18-22 May 2013, San Francisco, California, USA other psychiatric comorbidity, and the amount of in-patient care, affected mortality characteristics.

\section{Method}

\section{Study population}

The unique personal identity number assigned to each Swedish resident ${ }^{17}$ was used to link information from two populationbased registers, the National Patient Register and the Cause of Death Register. All individuals admitted to hospital in Sweden between 1987 and 2011 with a primary diagnosis of personality disorder were identified using the National Patient Register. This register includes all individuals admitted to any psychiatric or general hospital, has partial coverage between 1973 and 1986, and has almost complete coverage from $1987 .{ }^{18}$ Patients were classified according to ICD $-9^{19}$ between 1987 and 1996 and according to ICD-10 between 1997 and 2011, and grouped into clusters according to DSM-IV as follows: cluster A: paranoid (ICD-9 3010, ICD-10 F600), schizoid (ICD-9 3012, ICD-10 F601); cluster B: antisocial/dissocial (ICD-9 3017, ICD-10 F602), emotionally unstable/explosive/borderline (ICD-9 3013, ICD-10 F603), histrionic (ICD-9 3015, ICD-10 F604); cluster C: anankastic (ICD-9 3014, ICD-10 F605), anxious (avoidant) (ICD-9 N/A, ICD-10 F606), dependent (ICD-9 3016, ICD-10 F607); and unclustered: other specific (ICD-9 3018, ICD-10 F608) and unspecified (ICD-9 3019, ICD-10 F609). Patients not included in cluster A, B or C are classified as 'other personality disorder'.

Initially, all individuals admitted to hospital with a primary diagnosis of personality disorder were included $(n=27329)$. We subsequently identified those aged 15-64 at the time of their first admission to hospital ( $n=25726)$. After excluding 2388 individuals with a prior admission to hospital for personality disorder before 
1987, our final cohort comprised 23338 individuals. Data on admissions to hospital with somatic and psychiatric primary diagnoses other than personality disorder were also obtained. The Cause of Death Register was used to obtain information on cause of death. This register contains information on all deceased Swedish residents since 1952. The validity is high, and cause of death is lacking in only $0.5 \%$ of the deceased. ${ }^{20}$ The patients were followed from the date of first discharge until death or until the end of the follow-up period (i.e. 31 December 2011). Classification of personality disorders as well as underlying causes of death were coded according to ICD-9 between 1987 and 1996 and according to ICD-10 between 1997 and 2011. The study was approved by the Regional Ethical Review Board in Stockholm, protocol 2013/2028-31/5.

\section{Statistical analysis}

The number of expected deaths was calculated by multiplying the number of person-years at risk by 5 -year age group and calendaryear specific mortality rates in the general population. The SMR, i.e. the ratio between the observed number of deaths and the expected number of deaths during the follow-up period, was used as a measure of risk. The SMRs were calculated on the basis of 282188 person-years with 95\% confidence intervals assuming that the observed number of deaths in each group followed a Poisson distribution. Expected survival probabilities were calculated from general population life tables. We used SAS v. 9.2 for analysis.

\section{Results}

In total, 13740 women and 9598 men with a mean age slightly over 30 years were admitted to hospital with a primary diagnosis of personality disorder at least once between 1987 and 2011 (Table 1). Out of those, 1498 women and 1793 men, $11 \%$ and $19 \%$ of the cohort, respectively, died during the follow-up period. The most common diagnosis in women (49\%) was a cluster B personality disorder, whereas the most common diagnosis in men $(58 \%)$ was 'other personality disorder'. Together these diagnostic groups represented $93 \%$ and $86 \%$, respectively, of the personality disorder diagnoses. Comorbidity was common, as over $90 \%$ of both women and men received hospital care for concurrent psychiatric or somatic diseases during or after the first admission for personality disorder.

The SMRs for all those with personality disorder and by the different clusters are presented for women and men, respectively, in Tables 2 and 3. The all-cause SMRs were 6.1 (95\% CI 5.8-6.4) for women and 5.0 (95\% CI 4.7-5.2) for men (Tables 2 and 3). About half of the deaths were because of natural causes. The most common natural cause of death in women was cardiovascular disease at $17 \%$, followed by cancer at $13 \%$. In men, cardiovascular disease was also the most common natural cause of death at $21 \%$, followed by deaths with undetermined intent at $8 \%$ and cancer at $8 \%$. The highest SMRs for natural causes in women were observed for mental disorders: for substance misuse it was 11.4 (95\% CI 7.2-18.2) and for other mental disorders it was 13.3 (95\% CI 9.2-19.3). Besides that, there was a general increase in SMR for most somatic diagnostic groups, both in women and in men. Notably, the SMR for infections was as high as 8.6 (95\% CI 5.6-13.2) in women and 10.7 (95\% CI 7.8-14.7) in men, and for respiratory disorders it was 7.9 (95\% CI 6.3-9.9) in women and 5.5 (95\% CI 4.2-7.0) in men. Close to half of the deaths were as a result of unnatural causes, resulting in a considerably increased SMR of 22.0 (95\% CI 20.4-23.6) in women and 11.4 (95\% CI 10.6-12.1) in men. The highest SMRs for both genders were observed for suicide: 32.8 (95\% CI 30.0-35.8) in women and 16.4 (95\% CI 15.1-17.9) in men. Suicide was also the most common cause of death in both genders: $34 \%$ in women and $28 \%$ in men. We also confirmed recent observations of an increased risk for homicide, ${ }^{10}$ with SMRs of 5.6 (95\% CI $2.8-11.3)$ in women and 4.7 (95\% CI 2.7-8.3) in men.

Table 1 Descriptive statistics for all patients admitted to hospital for a personality disorder between 1987 and 2011 in Sweden

\begin{tabular}{|c|c|c|c|c|}
\hline & \multicolumn{2}{|c|}{ Women } & \multicolumn{2}{|c|}{ Men } \\
\hline & Total $(n=13740)$ & Deceased $(n=1498)$ & Total $(n=9598)$ & Deceased $(n=1793)$ \\
\hline Age, years: mean (s.d.) & $31.0(11.4)$ & & $32.6(11.1)$ & \\
\hline \multicolumn{5}{|l|}{ Age at first admission to hospital, years: $n(\%)$} \\
\hline $15-19$ & 2032 (15) & $90(6)$ & $658(7)$ & $63(4)$ \\
\hline $20-24$ & $3169(23)$ & $175(12)$ & $1643(17)$ & $196(11)$ \\
\hline $25-34$ & $4003(29)$ & $321(21)$ & 3299 (34) & $479(27)$ \\
\hline $35-44$ & 2516 (18) & $384(26)$ & $2224(23)$ & $440(25)$ \\
\hline $45-54$ & $1444(11)$ & $299(20)$ & $1284(13)$ & $372(21)$ \\
\hline $55-64$ & $576(4)$ & $229(15)$ & $490(5)$ & $243(14)$ \\
\hline \multicolumn{5}{|l|}{ Diagnostic group, n (\%) } \\
\hline Cluster A & 547 (4) & $86(6)$ & 1025 (11) & $210(12)$ \\
\hline Cluster B & 6754 (49) & $582(39)$ & $2658(28)$ & $459(26)$ \\
\hline Cluster C & 457 (3) & $65(4)$ & $367(4)$ & $66(4)$ \\
\hline Other & $5982(44)$ & $765(51)$ & $5548(58)$ & 1058 (59) \\
\hline Other specific personality disorder & $1098(8)$ & $169(11)$ & 1099 (11) & 230 (13) \\
\hline Personality disorder, unspecified & $4884(36)$ & $596(40)$ & $4449(46)$ & $828(46)$ \\
\hline \multicolumn{5}{|l|}{ Comorbidity during follow-up, $n$ (\%) } \\
\hline None & $361(3)$ & $24(2)$ & $641(7)$ & $59(3)$ \\
\hline Psychiatric & $766(6)$ & 74 (5) & 1099 (11) & $157(9)$ \\
\hline Somatic & $2130(16)$ & $194(13)$ & $1459(15)$ & $224(12)$ \\
\hline Both & $10483(76)$ & $1206(81)$ & $6399(67)$ & $1353(75)$ \\
\hline \multicolumn{5}{|l|}{ Years from first discharge to death, $n(\%)$} \\
\hline$<1$ & & $202(13)$ & & 244 (14) \\
\hline $1-2$ & & 199 (13) & & 275 (15) \\
\hline $3-4$ & & $169(11)$ & & $211(12)$ \\
\hline $5-9$ & & $323(22)$ & & $396(22)$ \\
\hline 10-19 & & 520 (35) & & $562(31)$ \\
\hline $20+$ & & $85(6)$ & & 105 (6) \\
\hline
\end{tabular}




\begin{tabular}{|c|c|c|c|c|c|}
\hline \multirow[b]{2}{*}{ Cause of death } & \multicolumn{5}{|c|}{ Standardised mortality ratios $(95 \% \mathrm{Cl})$} \\
\hline & All & Cluster A & Cluster B & Cluster C & Other \\
\hline Total & $6.1(5.8-6.4)$ & $4.3(3.5-5.3)$ & $6.4(5.9-7.0)$ & $5.0(4.0-6.4)$ & $6.3(5.9-6.8)$ \\
\hline Natural & $3.6(3.4-3.9)$ & $3.4(2.7-4.4)$ & $3.4(3.0-3.8)$ & $3.7(2.7-5.0)$ & $3.8(3.5-4.2)$ \\
\hline Infections & $8.6(5.6-13.2)$ & $5.3(0.8-37.8)$ & $2.2(0.5-8.7)$ & $8.2(1.2-58.3)$ & $14.1(8.7-22.6)$ \\
\hline Cancer & $1.6(1.4-1.9)$ & $2.4(1.6-3.5)$ & $1.9(1.5-2.3)$ & $1.4(0.7-2.6)$ & $1.3(1.1-1.7)$ \\
\hline Endocrine & $6.8(4.8-9.6)$ & $2.6(0.4-18.1)$ & $3.9(1.8-8.1)$ & $12.0(3.9-37.1)$ & $9.1(6.0-13.9)$ \\
\hline \multicolumn{6}{|l|}{ Mental } \\
\hline Substance misuse & $11.4(7.2-18.2)$ & - & $15.1(7.8-28.9)$ & $13.3(1.9-94.7)$ & $10.2(5.1-20.4)$ \\
\hline Other & $13.3(9.2-19.3)$ & $14.1(3.5-56.3)$ & $9.7(4.9-19.4)$ & $32.3(10.4-100)$ & $14.4(8.7-23.9)$ \\
\hline Nervous system & $4.9(3.5-6.7)$ & $1.7(0.2-12.1)$ & $4.2(2.4-7.3)$ & $2.6(0.4-18.7)$ & $6.1(4.1-9.2)$ \\
\hline Cardiovascular & $5.9(5.2-6.6)$ & $5.0(3.2-7.8)$ & $5.0(3.9-6.2)$ & $7.5(4.8-11.7)$ & $6.5(5.5-7.7)$ \\
\hline Respiratory & $7.9(6.3-9.9)$ & $9.3(4.7-18.7)$ & $8.6(5.9-12.4)$ & $5.5(1.8-17.1)$ & $7.4(5.3-10.4)$ \\
\hline Gastrointestinal & $4.9(3.6-6.6)$ & $3.9(1.3-12.2)$ & $3.1(1.7-5.8)$ & $4.2(1.0-16.6)$ & $6.4(4.4-9.3)$ \\
\hline Other & $6.1(4.8-7.7)$ & $2.5(0.6-10.1)$ & $6.3(4.3-9.2)$ & $1.9(0.3-13.8)$ & $6.8(4.9-9.4)$ \\
\hline Unnatural & $22.0(20.4-23.6)$ & $12.8(8.5-19.3)$ & $23.2(20.8-25.9)$ & $18.0(11.8-27.3)$ & $22.2(20.1-24.7)$ \\
\hline Suicide & $32.8(30.0-35.8)$ & $21.5(13.4-34.7)$ & $34.5(30.2-39.3)$ & $25.8(15.3-43.5)$ & $33.0(29.1-37.3)$ \\
\hline Undetermined intent & $22.3(18.5-26.9)$ & $7.2(1.8-28.7)$ & $26.4(20.2-34.6)$ & $16.0(5.2-49.7)$ & $21.2(16.1-27.8)$ \\
\hline Homicide & $5.6(2.8-11.3)$ & - & $3.2(0.8-12.8)$ & & $8.7(3.9-19.4)$ \\
\hline Traffic accidents & $2.8(1.7-4.6)$ & - & $3.0(1.4-6.3)$ & $5.5(0.8-38.8)$ & $2.7(1.3-5.6)$ \\
\hline Other & $15.2(12.4-18.6)$ & $9.9(3.7-26.3)$ & $15.1(10.9-20.8)$ & $15.0(5.6-39.9)$ & $16.1(12.2-21.3)$ \\
\hline
\end{tabular}

\begin{tabular}{|c|c|c|c|c|c|}
\hline \multirow[b]{2}{*}{ Cause of death } & \multicolumn{5}{|c|}{ Standardised mortality ratios $(95 \% \mathrm{Cl})$} \\
\hline & All & Cluster A & Cluster B & Cluster C & Other \\
\hline Total & $5.0(4.7-5.2)$ & $4.1(3.6-4.7)$ & $5.6(5.2-6.2)$ & $3.2(2.5-4.1)$ & $5.1(4.8-5.4)$ \\
\hline Natural & $3.2(3.0-3.5)$ & $3.1(2.6-3.6)$ & $3.2(2.8-3.7)$ & $2.7(2.0-3.5)$ & $3.3(3.1-3.6)$ \\
\hline Infections & $10.7(7.8-14.7)$ & $4.0(1.0-15.9)$ & $11.8(6.4-21.9)$ & $5.2(0.7-36.8)$ & $12.4(8.4-18.2)$ \\
\hline Cancer & $1.5(1.3-1.7)$ & $1.5(1.0-2.3)$ & $1.3(0.9-1.9)$ & $1.1(0.5-2.4)$ & $1.6(1.3-1.9)$ \\
\hline Endocrine & $4.0(2.8-5.6)$ & $2.6(0.8-8.1)$ & $2.2(0.8-5.9)$ & $10.9(4.5-26.2)$ & $4.3(2.8-6.7)$ \\
\hline \multicolumn{6}{|l|}{ Mental } \\
\hline Substance misuse & $7.3(5.5-9.7)$ & $3.3(1.1-10.2)$ & $8.2(4.8-14.1)$ & - & $8.5(6.1-12.0)$ \\
\hline Other & $5.4(3.9-7.6)$ & $5.2(1.9-13.8)$ & $6.3(3.4-11.6)$ & - & $5.6(3.6-8.6)$ \\
\hline Nervous system & $5.1(3.8-6.9)$ & $6.1(2.9-12.8)$ & $3.9(2.0-7.8)$ & $4.5(1.1-18.0)$ & $5.5(3.7-7.9)$ \\
\hline Cardiovascular & $3.4(3.1-3.8)$ & $3.7(2.8-4.7)$ & $3.4(2.7-4.3)$ & $3.2(2.1-4.8)$ & $3.4(2.9-3.8)$ \\
\hline Respiratory & $5.5(4.2-7.0)$ & $4.3(2.0-9.0)$ & $4.3(2.3-8.0)$ & $5.9(2.2-15.7)$ & $6.2(4.5-8.4)$ \\
\hline Gastrointestinal & $3.2(2.4-4.1)$ & $3.9(2.1-7.3)$ & $1.8(0.9-3.8)$ & $3.0(1.0-9.3)$ & $3.5(2.5-4.9)$ \\
\hline Other & $5.9(4.8-7.3)$ & $4.8(2.6-9.0)$ & $8.1(5.6-11.5)$ & $2.5(0.6-10.1)$ & $5.6(4.2-7.4)$ \\
\hline Unnatural & $11.4(10.6-12.1)$ & $9.1(7.3-11.4)$ & $12.7(11.3-14.4)$ & $6.4(4.2-10.0)$ & $11.5(10.5-12.5)$ \\
\hline Suicide & $16.4(15.1-17.9)$ & $15.7(12.1-20.5)$ & $16.0(13.6-19.0)$ & $12.3(7.4-20.3)$ & $17.0(15.2-19.1)$ \\
\hline Undetermined intent & $15.2(12.9-17.8)$ & $9.8(5.4-17.6)$ & $20.1(15.4-26.2)$ & $2.6(0.4-18.3)$ & $14.7(11.9-18.2)$ \\
\hline Homicide & $4.7(2.7-8.3)$ & - & $8.3(3.7-18.5)$ & - & $4.1(1.8-9.1)$ \\
\hline Traffic accidents & $3.4(2.5-4.5)$ & $2.2(0.7-6.7)$ & $4.8(3.0-7.7)$ & $2.0(0.3-14.3)$ & $3.0(2.0-4.5)$ \\
\hline Other & $7.7(6.6-9.0)$ & $4.5(2.5-8.0)$ & $9.8(7.4-12.9)$ & $3.3(1.1-10.2)$ & $7.9(6.4-9.7)$ \\
\hline
\end{tabular}

All three personality disorder clusters as well as 'other personality disorder' exhibited an increased SMR in both genders (Tables 2 and 3), although those with cluster B and 'other personality disorder' displayed somewhat higher SMRs than the other groups; cluster B all-cause mortality was 6.4 (95\% CI 5.97.0) in women and 5.6 (95\% CI 5.2-6.2) in men and 'other personality disorder' all-cause mortality was 6.3 (95\% CI 5.96.8) in women and 5.1 (95\% CI 4.8-5.4) in men. The SMR for suicide in those with a cluster B diagnosis was as high as 34.5 (95\% CI 30.2-39.3) in women and 16.0 (95\% CI 13.6-19.0) in men, and was similar among those with 'other personality disorder': 33.0 (95\% CI 29.1-37.3) in women and 17.0 (95\% CI 15.2-19.1) in men. Furthermore, death with undetermined intent was highest in those with a cluster B disorder; the SMR was 26.4 (95\% CI 20.2-34.6) in women and 20.1 (95\% CI 15.4-26.2) in men. Finally, all homicides occurred in those with cluster B or 'other personality disorder'.
In total $46 \%$ of women and $55 \%$ of men were admitted to hospital only once with a primary diagnosis of personality disorder (online Table DS1). In contrast, $9 \%$ of the women and $4 \%$ of the men had more than 10 admissions with that primary diagnosis. Most patients ( $82 \%$ of women and $78 \%$ of men) had also been admitted to hospital for other psychiatric disorders after their first admission for personality disorder, and also for somatic disorders ( $92 \%$ of women and $81 \%$ of men). There was already an increased SMR for all-cause mortality after one single admission period with a primary diagnosis of personality disorder: 5.3 (95\% CI 4.9-5.7) in women and 4.6 (95\% CI 4.3-4.9) in men. Those admitted to hospital more frequently had only a slight further increase in SMR, for example, 7.3 (95\% CI 6.2-8.6) in women and 6.7 (95\% CI 5.4-8.3) in men admitted more than 10 times. This result suggests that the number of admissions is not a severity marker per se for the overall risk for premature death. At the same time, the risk for unnatural death in women, 
particularly suicide, was considerably increased in women admitted more than once (online Fig. DS1). The increase in SMR remained stable after two or more admissions: 25.8 (95\% CI 22.2-29.9) in those admitted only once, 39.6 (95\% CI 32.5-48.2) in those admitted twice and 39.2 (95\% CI 30.7-50.0) in those admitted more than 10 times. In men, a similar but less prominent pattern was seen; the SMR for suicide was 15.0 (95\% CI 13.2-17.1) for those admitted once, it was highest for those admitted three times, 21.9 (95\% CI 17.1-28.1) and it was 16.9 (95\% CI 11.4-25.0) for those admitted more than 10 times.

Comorbidity had a negative effect on overall SMR. Thus, the SMR for all causes increased from 3.3 (95\% CI 2.2-4.9) in women and 2.4 (95\% CI 1.9-3.1) in men without any comorbidity, to 6.5 (95\% CI 6.1-6.9) in women and 5.7 (95\% CI 5.4-6.0) in men admitted with both psychiatric and somatic diagnoses (Tables 4 and 5). The effect of psychiatric comorbidity was most obvious for unnatural causes of death, and particularly for death with undetermined intent. A comparison between those without comorbidity and those with psychiatric comorbidity showed that the SMR for death with undetermined intent increased from 7.7 (95\% CI 1.1-55.0) to 32.9 (95\% CI 17.1-63.2) in women, and from 4.1 (95\% CI 1.3-12.6) to 18.8 (95\% CI 12.4-28.5) in men. The effect of somatic comorbidity was more obvious for natural causes of death and resulted in a slightly increased all-cause SMR. Finally, there was no increase in SMR for natural causes in women or men with personality disorder without any comorbidity (SMRs of 1.5 (95\% CI $0.8-2.9$ ) and 1.0 (95\% CI 0.6-1.6), respectively).

The increased SMR in individuals admitted to hospital for personality disorder is also reflected in a decreased cumulative survival over time (online Fig. DS2). The survival curves show a virtually linear decline in both women and men. Twenty years after their first discharge, $35 \%$ of the women and $41 \%$ of the men aged 40-64 at their first admission were dead, compared with an expected $10 \%$ and $15 \%$, respectively. In those aged 15-39 at their first admission corresponding figures were $11 \%$ and $18 \%$ $v$. an expected $2 \%$ and $3 \%$, respectively.

\section{Discussion}

\section{Main findings}

Our study shows that people who have been admitted to hospital with a diagnosis of personality disorder have a substantially increased risk of death compared with the general population, with five- to six-fold increased all-cause mortality, three-fold increased mortality for natural causes and more than 10-fold increased mortality for unnatural causes. Furthermore, in those with a comorbid psychiatric disorder the risk for suicide was found to be increased as much as 42-fold in women and 19-fold in men. The core results validate those found in a previous investigation in a smaller sample of people with personality disorder who had been treated as in-patients in northern Norway. ${ }^{9}$ The most important finding is that the increased mortality is distributed among all clusters of personality disorder, and that differences between clusters are much smaller than the difference between the general population and people with personality disorder. There are virtually no differences between clusters with respect to natural deaths, whereas unnatural deaths are somewhat more frequent in cluster B and in 'other personality disorder' than in clusters $\mathrm{A}$ and $\mathrm{C}$.

\section{Comparison with findings from other studies}

The gender distribution and the distribution between clusters in this cohort of patients with hospital admissions for personality disorder are similar to those in previous studies, i.e. with about $60 \%$ women and the dominance of cluster B and 'other personality disorder.' These findings differ from global health survey data, where the incidence of personality disorder in males is about 2.5 times as high as in females, and with a fairly equal distribution between clusters. ${ }^{1}$ A possible explanation is that more cluster B patients receive care than those with clusters $\mathrm{A}$ and $\mathrm{C},{ }^{12}$ because, logically, of the clinical characteristics of cluster B, and also a gender bias when making psychiatric diagnoses. ${ }^{21}$ Furthermore, most patients in clinical practice with severe personality pathology do not fulfil criteria for a specified personality disorder diagnosis. ${ }^{21}$ This issue has been discussed previously in the context of findings in the National Comorbidity Survey Replication (NCS-R). ${ }^{12}$ There is also reason to suppose that patients reported as having 'other personality disorder' have more severe personality pathology, ${ }^{22}$ as standard personality prototypes tend to merge as disturbance gets more severe ${ }^{23}$ and that greater severity is associated with greater mortality, including suicidal behaviour. ${ }^{24}$

The all-cause mortality in in-patient-treated individuals with a diagnosis of personality disorder is similar to that reported previously, ${ }^{6,7,25}$ and is also similar, for example, to that reported for anorexia nervosa (an SMR of 6.1 in women in the present study and 6.2 in patients with anorexia nervosa ${ }^{26}$ ). It is considerably higher than that found previously for both affective disorders, with SMRs of 2.5 for bipolar disorders and 2.0 for unipolar disorders, ${ }^{27}$ and for schizophrenia, with an SMR of 2.8. ${ }^{28}$ Comparisons with these studies are appropriate as data are based on the same background population, using the same healthcare system and national healthcare registers.

\section{Deaths by suicide}

It is well established that patients with cluster B personality disorder, particularly borderline personality disorder and antisocial personality disorder, have an increased risk for suicide. $^{5,9,29,30}$ Thus, an accepted truth has been that about $8-10 \%$ of those receiving a diagnosis of borderline personality disorder die by suicide, ${ }^{31}$ which is about 50 times more than in the general population, ${ }^{32}$ although SMR data for larger patient cohorts are lacking in the literature. We show (Tables 2 and 3 ) a mortality rate for suicide in women admitted to hospital for cluster B personality disorder that is close to the previously mentioned figure, an SMR of 34.5 (95\% CI 30.2-39.3), whereas in men the figure is lower, SMR 16.0 (95\% CI 13.6-19.0). Not unexpectedly, the high suicide mortality within cluster B is seen in those with a borderline personality disorder, as a subgroup analysis showed that the mortality from suicide in those with a diagnosis of borderline personality disorder was higher, although marginally so, than that in the aggregated cluster B group, both in women and in men (online Table DS3). A striking observation is that the mortality from suicide was similar in those with cluster B personality disorder and in those with 'other personality disorder', and also almost as high in those with cluster A and cluster $\mathrm{C}$ personality disorder. We also noticed that being admitted to hospital twice or more was associated with a further increased suicide rate in women. This is in accordance with the concept that women die by suicide after a number of attempts whereas men often take their own life at their first attempt. ${ }^{33}$

\section{Deaths from natural causes}

The increased mortality because of natural causes in patients with personality disorder has been explained by poorer general health and an unhealthy lifestyle, ${ }^{6}$ and also by barriers to receiving 

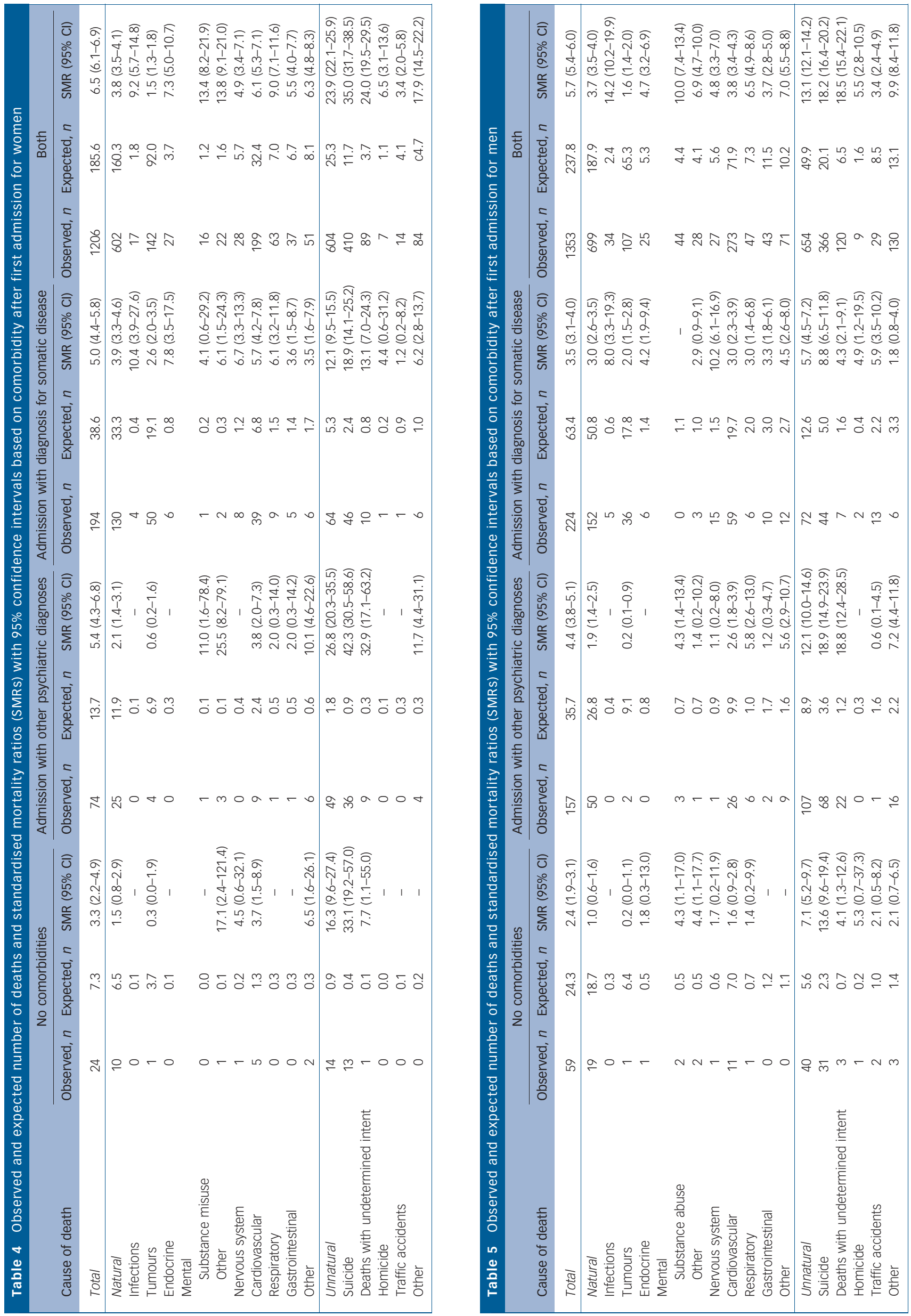
and maintaining adequate care for their comorbid physical conditions. ${ }^{34,35}$ It is noteworthy that patients with personality disorder without any registered somatic or psychiatric comorbidity did not present with an increased SMR for natural causes.

\section{Psychiatric comorbidity}

Psychiatric comorbidity had only a moderate potentiating effect on the SMR for unnatural causes. This is consistent with previous studies suggesting that patients with personality disorder seek healthcare for their comorbid Axis I disorders, although their impairments are not a result of these disorders. ${ }^{1}$ The contribution of psychiatric comorbidity to the increased risk for premature death from unnatural causes is also in accordance with previous data indicating that patients with personality disorder who die by suicide commonly have a comorbid diagnosis, most often substance misuse or depressive disorder. ${ }^{36}$

\section{Strengths and limitations}

The strengths of the study include the population-based design using national registers with high levels of completeness and validity. However, there are some weaknesses. First, the study is based on patients with hospital admissions. Hospital admissions account for only a small part of all psychiatric interventions in Sweden, as in most countries. There is a true risk of selection bias in that a subgroup of patients with more severe problems in personality functioning are overrepresented in our cohort. Therefore, uncritical extrapolation to patients with personality disorder who are not treated as in-patients is not possible. On the other hand, a study based on no fewer than 1095338 Swedish men conscripted to military service between 1969 and 1994 showed that the increased risk of premature death associated with psychiatric disorders in general is not confined to those who have been admitted to hospital. ${ }^{25}$ Furthermore, in a first analysis of patients in out-patient care in Sweden, with data from 2001 to 2011, mortality was also increased in those given only an out-patient diagnosis of personality disorder (data not shown).

Another issue is that the Swedish healthcare system requires that each patient be given a formal diagnosis. This is done at the discretion of the treating physician, which is why its scientific validity in individual cases can be questioned. Furthermore, the Swedish National Patient Register is based on the ICD diagnostic system, whereas the personality disorder cluster concept is part of the DSM system. As the diagnostic concordance between the systems is acceptable to high with respect to different personality disorders ${ }^{37}$ we chose to apply the cluster concept in the present study. This was also motivated by the fact that in spite of the relatively large cohort there was not sufficient statistical power to report the findings for each personality disorder by primary ICD code/category. Moreover, there is not total agreement between ICD-9 and ICD-10 with respect to the criteria for different personality disorders. This has an impact on the criteria for cluster B and 'other personality disorder' that might influence interpretation of the data. We therefore analysed subcohorts with ICD-9 and ICD-10 data separately, and adjusted the follow-up time for the former to fit with that of the latter. Both cohorts showed similar SMRs, particularly in cluster B and 'other personality disorder', although with very wide confidence intervals for the less common clusters A and C (online Table DS2). It must also be kept in mind that certain individual behaviours indicating a risk of mortality, such as suicidality or excessive risk-taking, may constitute an incentive to consider a diagnosis of personality disorder.
An additional issue is that we were not able to control for known potentially important confounders such as family history of personality disorder or other mental disorders, or for socioeconomic status. Finally, the study design, which was chosen to maximise time for follow-up and thereby increase power in the analysis, did not allow for an assessment of in-patient care of other causes than personality disorder before the first-index episode. Morbidity was therefore only based on in-patient care occurring after the first episode. A consequence was that the time course of symptoms leading to in-patient care, and the importance of these with respect to mortality, could not be studied.

\section{Implications}

Patients with a personality disorder diagnosis consume a great deal of healthcare as a result of physical as well as mental conditions. ${ }^{38,39}$ Despite higher levels of healthcare utilisation, they have a profoundly increased mortality risk. This marked increase in excess mortality casts a shadow over the issue of whether they are given the care they need. ${ }^{7}$ Increased awareness on the part of professionals is desirable regarding the health risks associated with personality disorder.

\section{Funding}

E.B. and C.B. were employed by the Swedish National Board of Health and Welfare during the study. Additional financial support was provided by a grant to L.E. from the Swedish Research Council.

Emma Björkenstam, PhD, Department of Public Health Sciences, Karolinska Institutet, Stockholm and the Department of Evaluation, and Analysis, Epidemiology and Methodological Support Unit, National Board of Health and Welfare, Stockholm, Sweden; Charlotte Björkenstam, PhD, Department of Clinical Neuroscience, Karolinska Institutet, Stockholm, Sweden; Herman Holm, MD, Department of Psychiatry, Skåne University Hospital, Malmö, Sweden; Bengt Gerdin, MD, PhD, Department of Surgical Sciences, Uppsala University, Uppsala, Sweden; Lisa Ekselius, MD, PhD, Department of Neuroscience, Psychiatry, Uppsala University,
Ex Uppsala, Sweden

Correspondence: Lisa Ekselius, MD, PhD, Department of Neuroscience, Psychiatry, Uppsala University, Uppsala University Hospital, SE-751 85 Uppsala, sweden. Email: lisa.ekselius@neuro.uu.se

First received 8 Apr 2014, final revision 26 Nov 2014, accepted 27 Nov 2014

\section{References}

1 Huang $Y$, Kotov R, de Girolamo G, Preti A, Angermeyer M, Benjet C, et al. DSM-IV personality disorders in the WHO World Mental Health Surveys. Br J Psychiatry 2009; 195: 46-53.

2 American Psychiatric Association. Diagnostic and Statistical Manual of Mental Disorders (4th edn, revised) (DSM-IV-TR). APA, 2000.

3 World Health Organization. The ICD-10 Classification of Mental and Behavioural Disorders: Clinical Descriptions and Diagnostic Guidelines. WHO, 1992.

4 Harris EC, Barraclough B. Excess mortality of mental disorder. Br J Psychiatry 1998; 173: 11-53.

5 Grigoletti L, Perini G, Rossi A, Biggeri A, Barbui C, Tansella M, et al. Mortality and cause of death among psychiatric patients: a 20-year case-register study in an area with a community-based system of care. Psychol Med 2009; 39: 1875-84.

6 Fok ML, Hayes RD, Chang CK, Stewart R, Callard FJ, Moran P. Life expectancy at birth and all-cause mortality among people with personality disorder. J PSychosom Res 2012; 73: 104-7.

7 Nordentoft M, Wahlbeck K, Hallgren J, Westman J, Osby U, Alinaghizadeh H, et al. Excess mortality, causes of death and life expectancy in 270,770 patients with recent onset of mental disorders in Denmark, Finland and Sweden. PLOS One 2013; 8: e55176.

8 Ajetunmobi O, Taylor M, Stockton D, Wood R. Early death in those previously hospitalised for mental healthcare in Scotland: a nationwide cohort study, 1986-2010. BMJ Open 2013; 3: e002768. 
9 Hoye A, Jacobsen BK, Hansen V. Sex differences in mortality of admitted patients with personality disorders in North Norway-a prospective register study. BMC Psychiatry 2013; 13: 317.

10 Crump C, Sundquist K, Winkleby MA, Sundquist K. Mental disorders and vulnerability to homicidal death: Swedish nationwide cohort study. BMJ 2013; 346: 5557.

11 Hiroeh U, Appleby L, Mortensen PB, Dunn G. Death by homicide, suicide, and other unnatural causes in people with mental illness: a population-based study. Lancet 2001; 358: 2110-2.

12 Lenzenweger MF, Lane MC, Loranger AW, Kessler RC. DSM-IV personality disorders in the National Comorbidity Survey Replication. Biol Psychiatry 2007; 62: 553-64.

13 Zimmerman $\mathrm{M}$, Chelminski I, Young $\mathrm{D}$. The frequency of personality disorders in psychiatric patients. Psychiatr Clin North Am 2008; 31: 405-20, vi.

14 Douzenis A, Tsopelas C, Tzeferakos G. Medical comorbidity of cluster B personality disorders. Curr Opin Psychiatry 2012; 25: 398-404.

15 Lee HB, Bienvenu OJ, Cho SJ, Ramsey CM, Bandeen-Roche K, Eaton WW, et al. Personality disorders and traits as predictors of incident cardiovascular disease: findings from the 23-year follow-up of the Baltimore ECA study Psychosomatics 2010; 51: 289-96.

16 Schneider B, Wetterling T, Sargk D, Schneider F, Schnabel A, Maurer K, et al. Axis I disorders and personality disorders as risk factors for suicide. Eur Arch Psychiatry Clin Neurosci 2006; 256: 17-27.

17 Ludvigsson JF, Otterblad-Olausson P, Pettersson BU, Ekbom A. The Swedish personal identity number: possibilities and pitfalls in healthcare and medical research. Eur J Epidemiol 2009; 24: 659-67.

18 Ludvigsson JF, Andersson E, Ekbom A, Feychting M, Kim JL, Reuterwall C, et al. External review and validation of the Swedish national inpatient register. BMC Public Health 2011; 11: 450.

19 World Health Organization. International Statistical Classification of Diseases and Related Health Problems (ICD-9). WHO, 1978.

20 National Board of Health and Welfare. Causes of Death 2011. National Board of Health and Welfare, 2012.

21 Flanagan EH, Blashfield RK. Gender bias in the diagnosis of personality disorders: the roles of base rates and social stereotypes. J Pers Disord 2003; 17: 431-46.

22 Verheul R, Bartak A, Widiger T. Prevalence and construct validity of personality disorder not otherwise specified (PDNOS). J Pers Disord 2007; 21 359-70.

23 Yang M, Coid J, Tyrer P. Personality pathology recorded by severity: national survey. Br J Psychiatry 2010; 197: 193-9.

24 Blasco-Fontecilla $\mathrm{H}$, Baca-Garcia E, Dervic K, Perez-Rodriguez MM, Saiz-Gonzalez MD, Saiz-Ruiz J, et al. Severity of personality disorders and suicide attempt. Acta Psychiatr Scand 2009; 119: 149-55.
25 Gale CR, Batty GD, Osborn DP, Tynelius P, Whitley E, Rasmussen F. Association of mental disorders in early adulthood and later psychiatric hospital admissions and mortality in a cohort study of more than 1 million men. Arch Gen Psychiatry 2012; 69: 823-31.

26 Papadopoulos FC, Ekbom A, Brandt L, Ekselius L. Excess mortality, causes of death and prognostic factors in anorexia nervosa. Br J Psychiatry 2009; 194: 10-7.

27 Osby U, Brandt L, Correia N, Ekbom A, Sparen P. Excess mortality in bipolar and unipolar disorder in Sweden. Arch Gen Psychiatry 2001; 58: 844-50.

28 Osby U, Correia N, Brandt L, Ekbom A, Sparen P. Mortality and causes of death in schizophrenia in Stockholm county, Sweden. Schizophr Res 2000 45: 21-8.

29 Black DW, Blum N, Pfohl B, Hale N. Suicidal behavior in borderline personality disorder: prevalence, risk factors, prediction, and prevention. J Pers Disord 2004; 18: 226-39.

30 Goodman M, Roiff T, Oakes $\mathrm{AH}$, Paris J. Suicidal risk and management in borderline personality disorder. Curr Psychiatry Rep 2012; 14: 79-85.

31 Gunderson JG. Clinical practice. Borderline personality disorder. N Engl J Med 2011; 364: 2037-42.

32 Work Group on Borderline Personality Disorder. Practice guideline for the treatment of patients with borderline personality disorder. Am J Psychiatry 2001; 158 (suppl 10): 1-52

33 Moscicki EK. Gender differences in completed and attempted suicides. Ann Epidemiol 1994; 4: 152-8.

34 De Hert M, Cohen D, Bobes J, Cetkovich-Bakmas M, Leucht S, Ndetei DM, et al. Physical illness in patients with severe mental disorders. II. Barriers to care, monitoring and treatment guidelines, plus recommendations at the system and individual level. World Psychiatry 2011; 10: 138-51.

35 De Hert M, Correll CU, Bobes J, Cetkovich-Bakmas M, Cohen D, Asai I, et al. Physical illness in patients with severe mental disorders. I. Prevalence, impact of medications and disparities in health care. World Psychiatry 2011; 10: $52-77$.

36 Hunt IM, Kapur N, Robinson J, Shaw J, Flynn S, Bailey H, et al. Suicide within 12 months of mental health service contact in different age and diagnostic groups: National clinical survey. Br J Psychiatry 2006; 188: 135-42.

37 Ottosson H, Ekselius L, Grann M, Kullgren G. Cross-system concordance of personality disorder diagnoses of DSM-IV and diagnostic criteria for research of ICD-10. J Pers Disord 2002; 16: 283-92.

38 Coid J, Yang $M$, Tyrer $P$, Roberts A, Ullrich S. Prevalence and correlates of personality disorder in Great Britain. Br J Psychiatry 2006; 188: 423-31.

39 Torgersen S, Kringlen E, Cramer V. The prevalence of personality disorders in a community sample. Arch Gen Psychiatry 2001; 58: 590-6. 